Título artículo / Títol article: Persistent phagocytic characteristics of microglia in the substantia nigra of long-term Parkinsonian macaques

Autores / Autors

Revista:

Versión / Versió:

Cita bibliográfica / Cita bibliogràfica (ISO 690):
Carlos Barcia, Carmen María Ros, Francisco Ros-Bernal, Aurora Gómez, Valentina Annese, María Angeles Carrillo-de Sauvage, José Enrique Yuste, Carmen María Campuzano, Vicente de Pablos, Emiliano Fernández-Villalba, María Trinidad Herrero

Journal of Neuroimmunology (2013), Vol. 261, No. 1

\section{Postprint del autor}

BARCIA, Carlos; ROS, Carmen María; ROSBERNAL, Francisco; GÓMEZ, Aurora; ANNESE, Valentina; CARRILLO-DE SAUVAGE, María Ángeles; YUSTE, José Enrique; CAMPUZANO, Carmen María; PABLOS, Vicente de; FERNÁNDEZ-VILLALBA, Emiliano; HERRERO, María Trinidad. Persistent phagocytic characteristics of microglia in the substantia nigra of long-term Parkinsonian macaques . Journal of Neuroimmunology, 2013, vol. 261, no. 1, p. 60-66. 


\title{
Persistent phagocytic characteristics of microglia in the substantia nigra of long-term Parkinsonian macaques
}

\author{
Carlos Barcia ${ }^{\mathrm{a}, \mathrm{b}, *}$, Carmen María Ros ${ }^{\mathrm{a}, \mathrm{b}}$, Francisco Ros-Bernal ${ }^{\mathrm{a}, \mathrm{b}}$, Aurora Gómez ${ }^{\mathrm{a}, \mathrm{b}}$, Valentina Annese ${ }^{\mathrm{a}, \mathrm{b}}$, \\ María Angeles Carrillo-de Sauvage ${ }^{\mathrm{a}, \mathrm{b}}$, José Enrique Yuste ${ }^{\mathrm{a}, \mathrm{b}}$, Carmen María Campuzano a , \\ Vicente de Pablos ${ }^{\mathrm{a}, \mathrm{b}}$, Emiliano Fernández-Villalba ${ }^{\mathrm{a}, \mathrm{b}}$, María Trinidad Herrero ${ }^{\mathrm{a}, \mathrm{b}}$ \\ a Clinical and Experimental Neuroscience, Spain

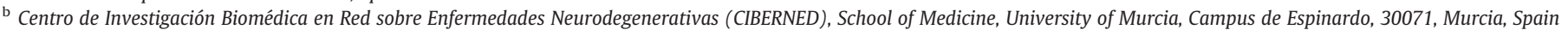

\section{A R T I C L E I N F O}

\section{Article history:}

Received 12 December 2012

Received in revised form 3 May 2013

Accepted 9 May 2013

Available online $\mathrm{xxxx}$

\section{Keywords:}

Parkinson's disease

MPTP

Dopaminergic degeneration

Cell polarity

Inflammation

Microglial motility

\begin{abstract}
A B S T R A C T
Patients with Parkinson's disease show persistent microglial activation in the areas of the brain where the degeneration of dopaminergic neurons takes place. The reason for maintaining this activated state is still unknown, but it is thought that this persistent microglial activation may contribute to the degeneration of dopaminergic neurons. In this study, we report the microanatomical details of microglia and the relationship between microglia and neurons in the substantia nigra pars compacta of Parkinsonian monkeys years after insult with MPTP. We observed that microglial cells appear polarized toward dopaminergic neurons in MPTP-treated macaques compared to untreated animals and present clear phagocytic characteristics, such as engulfing gliaptic contacts, an increase in Golgi apparatus protein machinery and ball-and-chain phagocytic buds. These results demonstrate that activated microglia maintain phagocytic characteristics years after neurotoxin insult, and phagocytosis may be a key contributor to the neurodegenerative process.
\end{abstract}

(c) 2013 Elsevier B.V. All rights reserved.

\section{Introduction}

Microglial activation is associated with the release of cytokines, an increase in particular membrane receptors, changes in morphology, and the self-induction of directional motility and polarity (Kreutzberg, 1996; Nimmerjahn et al., 2005; Barcia et al., 2011). The purpose of microglial activation in neurodegenerative diseases remains unclear. In Parkinson's disease (PD) patients, the areas of the brain that contain dopaminergic degeneration show high levels of activated microglia that are maintained for decades (McGeer et al., 1988). The same effect is observed in humans intoxicated with MPTP (Langston et al., 1999) as well as in long-term MPTP-induced Parkinsonian macaques (McGeer et al., 2003; Barcia et al., 2004). However, the specific role of microglia during this persistent stage is still under scrutiny. We and others have recently reported that in an experimental mouse model of PD in which Parkinsonism was induced by MPTP, microglial motility leads to the

\footnotetext{
Abbreviations: PD, Parkinson's disease; MPTP, 1-methyl-4-phenyl-1,2,3,6 tetrahydropyridine; SNpc, substantia nigra pars compacta; $\mathrm{TH}$, tyrosine hydroxylase; Iba-1, ionized calcium binding adaptor molecule 1; GM130, cis-Golgi matrix protein 130; DAPI, 4',6-diamidino-2-phenylindole.

* Corresponding author at: Clinical and Experimental Neuroscience and Centro de Investigación Biomedica en Red de Enfermedades Neurodegenerativas (CIBERNED), School of Medicine, University of Murcia, Campus de Espinardo, 30071 Murcia, Spain. Tel.: + 34609529 527; fax: + 34868884150 .

E-mail address: barcia@um.es (C. Barcia).
}

formation of complex body-to-body contacts between microglia and dopaminergic neurons that result in the phagocytosis of neuronal cell bodies in the areas of degeneration (Barcia et al., 2012; Depboylu et al., 2012). In mice, interactions between microglia and neurons are transient and precede the elimination of damaged dopaminergic neurons (Barcia et al., 2012).

To study the phagocytic characteristics of microglia in long-term Parkinsonism, we analyzed different parameters of microglial activation in the substantia nigra pars compacta (SNpc) in a group of macaques suffering from MPTP-induced Parkinsonism. We observed clear phagocytic features of microglia years after MPTP treatment, such as an increased number of contacts between microglia and dopaminergic neurons and the increase of phagocytic domains, as indicated by the increase of the Golgi apparatus protein machinery and ball-and-chain phagocytic buds. These characteristics are persistent years after MPTP treatment, which suggests that phagocytosis is an important aspect of microglial activation in chronic Parkinsonism that contributes to neurodegeneration.

\section{Material and methods}

\subsection{Parkinsonian monkeys}

In this study, we used a brain bank to obtain brain tissue samples from chronic Parkinsonian macaques (Macaca fascicularis). We studied 
these macaques throughout the years in our primate unit. All of the studies that were performed in primates before 2006 were carried out in accordance with the protocols from the states members of the European Union (2003/65/CE) and the National Institutes of Health Guide for the Care and Use of Laboratory Animals (NIH Guide, revised 1996). The experiments that were performed in monkeys after 2006 met the recommendations of the Weatherall report, the Guidelines promulgated by the European Convention for the protection of Vertebrate Animals used for Experimental and other scientific purposes of the Council of Europe (no. 123, June 15th, 2006), the European Communities Council Directive 2010/63/ECC, and the University of Murcia Approved protocols.

We analyzed samples obtained from young adult macaques from both sexes. Samples from eight animals were used in the present work. Four animals were treated with MPTP and four animals were untreated controls (Table 1 ). The monkeys were treated weekly with low doses of intravenous MPTP $(0.3 \mathrm{mg} / \mathrm{kg})$ according to previous protocols (Herrero et al., 1993; Barcia et al., 2003). Each animal received a different number of doses according to the level of Parkinsonism and their susceptibility to MPTP. None of the animals received L-DOPA or other dopaminergic agonists. Several researchers defined the degree of Parkinsonism in the animals after each dose as well as the progression of the Parkinsonian syndrome. Motor symptoms were assessed using a previously described rating scale ranging from 0 to 25 (Herrero et al., 1993). The degree of disability in the monkeys increased with every new injection and remained stable for months or years. Susceptible MPTP-treated monkeys showed Parkinsonian symptoms and displayed different degrees of bradykinesia and akinesia, and sporadic freezing phenomena. Some of the monkeys presented action tremor, paradoxical kinesia and occasionally vertical and horizontal saccadic ocular movements. Susceptible monkeys also displayed a typical Parkinsonian posture. The animals were euthanized one to 5 years after the last MPTP administration.

\subsection{Tissue staining}

The monkeys were euthanized by injection with pentobarbital after ketamine anesthesia one, two or five years after the last dose of MPTP. The brain was quickly removed, dissected and fixed for 3 days in $4 \%$ paraformaldehyde that was dissolved in $0.1 \mathrm{M}$ phosphate buffer. The fixed mesencephalon was sectioned into $40 \mu \mathrm{m}$ thick serial sections (Microm, HM400). Series of sections that were regularly spaced at intervals of $1440 \mu \mathrm{m}$ were stained for tyrosine hydroxylase (TH) (sheep polyclonal antibody 1:500; Chemicon, Temecula, CA, USA) to identify dopaminergic neurons, Iba-1 (rabbit polyclonal antibody 1:500; Wako, Chuo-Ku, Osaka, Japan) to identify microglial cells, and cis-Golgi Matrix protein 130 (GM130) (1:500; Abcam, Cambridge, UK) to detect the Golgi apparatus. Sections from all of the animals were stained simultaneously and under the same experimental conditions.

\subsubsection{Immunohistochemistry and immunofluorescence}

2.2.1.1. DAB detection. Sections of the SNpc ( $40 \mu \mathrm{m})$ were used to detect specific cells and structures via immunohistochemistry.

Table 1

Motor score and MPTP accumulative dose of used monkeys.

\begin{tabular}{llll}
\hline Monkey & Sex & Motor score & MPTP accumulative dose $\mathrm{mg} / \mathrm{kg}$ \\
\hline C1 & Male & 0.0 & 0 \\
C2 & Male & 0.0 & 0 \\
C3 & Female & 0.0 & 0 \\
C4 & Male & 0.0 & 0 \\
P1 & Male & 3.5 & 1.8 \\
P2 & Female & 4.2 & 0.9 \\
P3 & Male & 5.0 & 1.8 \\
P4 & Female & 9.2 & 0.9
\end{tabular}

Endogenous peroxidase activity was inhibited with $0.3 \% \mathrm{H}_{2} \mathrm{O}_{2}$, and non-specific Fc binding sites were blocked with $10 \%$ horse serum. The sections were incubated for $48 \mathrm{~h}$ (room temperature, constant shaking) with the primary antibody (see above) diluted in PBS containing $1 \%$ horse serum, $0.5 \%$ Triton $\mathrm{X}-100$, and $0.1 \%$ sodium azide. The sections were incubated for $4 \mathrm{~h}$ in the secondary antibody that was diluted in antibody solution. Binding of the antibody was detected with the avidin-biotin peroxidase ABC kit (Vectastain, Vector Labs). Sections were mounted on gelatin-coated slides and dehydrated in graded ethanol series and xylene before the addition of a coverslip.

2.2.1.2. Immunofluorescence. For immunofluorescence, $40 \mu \mathrm{m}$ sections were treated with $0.5 \%$ citrate buffer $\left(65^{\circ} \mathrm{C}\right.$, with constant shaking) for $30 \mathrm{~min}$ to maximize antibody penetration into the tissue. Nonspecific Fc binding sites were blocked with $10 \%$ horse serum, and the sections were incubated for $48 \mathrm{~h}$ (room temperature, constant shaking) with primary antibody diluted in PBS containing $1 \%$ horse serum, $0.5 \%$ Triton $\mathrm{X}-100$, and $0.1 \%$ sodium azide. The sections were incubated for $4 \mathrm{~h}$ with the labeled secondary antibody and were then washed with PBS and incubated with the DAPI solution (1:1000) in $1 \times$ PBS for $30 \mathrm{~min}$. The sections were washed, mounted, and examined using fluorescence (Zeiss Axioplan 2) or using confocal microscopy (Leica DMIRE2). The secondary antibodies used are as follows: Alexa 488conjugated, Alexa 594-conjugated or Alexa 647-conjugated (1:1000) (Molecular Probes). For F-actin staining, sections were incubated with Alexa Fluor 488-phalloidin or 647-phalloidin (1:500 in PBS; Molecular Probes) for $2 \mathrm{~h}$ at room temperature after immunostaining. After washing, the sections were incubated with a DAPI solution for $30 \mathrm{~min}$. The sections were washed again, mounted and examined by fluorescence (Zeiss, Axioplan 2) or confocal microscopy (DMIRE2, Leica Microsystems, Exton, PA).

2.2.1.3. Quantification and sterological analysis. The nigrostriatal pathway was defined according to the Monkey Brain Atlas (Paxinos et al., 1999). Quantification of DAB or fluorescently labeled cells in the SNpc was performed on coronal sections from each animal. The number of cells was estimated with an optical fractionator probe using a computer assisted image analysis system with a Zeiss Axioplan 2 microscope connected to a digital camera. The region of interest was traced using the $1.25 \times$ objective. The number of cells was measured in $200 \times 200 \mu \mathrm{m}$ dissectors covering the surface of the analyzed region. Labeled cells were counted using the $40 \times$ objective, and frames were counted throughout the delineated area of the SNpc in each section via the optical fractionator. Two representative sections of the SNpc were used for staining in monkey brains. Representative sections from the monkeys were chosen according to the criteria previously defined by Olzewski and Baxter (1954). The sections contained the ventral area of the SNpc (in primates corresponding to the $\alpha+\beta$ area at the level of the III cranial nerve output), which is known to show the highest degree of dopaminergic loss and glial activation in humans (Damier et al., 1999) and non-human primates (Herrero et al., 1993). The data are expressed as the absolute number of positive cells in each anatomical region analyzed. The results are expressed as the mean \pm SEM.

\section{Confocal analysis and 3D rendering}

The brain sections were examined using a Leica DMIRE2 confocal microscope with a $63 \times$ oil objective and Leica Confocal Software (Leica Microsystems). A series of optical sections were analyzed to determine an upper and lower threshold using the $\mathrm{Z} / \mathrm{Y}$ position for the Spatial Image Series setting. The optical series covered a $20 \mu \mathrm{m}$ thick slice of tissue with $0.5 \mu \mathrm{m}$ per optical section. The confocal microscope settings were established and maintained by Leica and local technicians for optimal resolution. (For further details, see previous publications (Barcia et al., 2011). Contacts were defined as areas where co-localization of both markers (Iba- 1 and TH) occurred between two 
cells in at least two $0.5-\mu m$-thick optical sections. A thin area of colocalization can be observed between the two fluorophores. The final confocal images are illustrated as they appear throughout the stack of sections as a simple 0.5 - $\mu$ m-thick layer or as a transparency of all of the layers merged together.

Three-dimensional reconstructions of the stacks of confocal images were rendered in the Imaris software (Bitplane Scientific Software. Zurich. Switzerland). Different surface effects were applied in the 3D rendering to show the structures in the real planes. Videos of the rotations were also generated with Imaris and were edited with iMovie Software (Apple Inc. Cupertino, CA) to obtain the final versions.

\subsection{Quantification of activated microglia phenotypes}

Sections of the SNpc from monkeys underwent immunofluorescence to visualize microglial cells (Iba- $1^{+}$cells). The sections were scanned with a confocal microscope as described above, and the terminal microglial tips per microglial cell were quantified along the $z$-axis. The microglial area and terminal tips were quantified using confocal images of the Iba- $1^{+}$cells. The scanned confocal planes with a thickness of $0.5 \mu \mathrm{m}$ that contain Iba- $1^{+}$cells were processed with the image software Image-J in order to obtain a black and white image. The area of 50 microglial cells (specified by Iba-1 staining) in the SNpc of each animal was measured in the processed images. Similarly, sections were costained for Iba-1 and GM130, and the area occupied by GM130 was quantified in Iba- $1^{+}$cells using the same method described for Iba- 1. Two different researchers did all of the quantifications blindly.

\subsection{Quantification of intercellular contacts}

Intercellular contacts between microglial cells and dopaminergic neurons or fibers were quantified using stereological methods in the confocal stacks of images of the SNpc. The number of contacts per field between cells that are positive for Iba- 1 and TH was quantified in $0.5 \mu \mathrm{m}$ single layers from the $20-\mu \mathrm{m}$-thick confocal stacks of images. The contacts between microglial cells and dopaminergic neurons were identified by the convergence of red and green fluorescence, which formed a thin yellow area. The contacts were classified into four categories: Pr-Pr, contact between a microglial process and a dopaminergic process; B-Pr, contact between a microglial cell body and a dopaminergic process; $\mathbf{P r}-\mathbf{B}$, contact between a microglial process and a dopaminergic neuron cell body; and B-B, contact between a microglial cell body and a dopaminergic neuron cell body. The number of contacts per DA fiber (Pr-Pr and B-Pr) was quantified using the total number of remaining fibers, which was quantified using unbiased stereological methods (Gundersen, 1986). The number of B-B and Pr-B contacts per neuron was quantified for each neuron along the $z$ plane. The length of the contacts between processes and neurons was quantified using Leica Confocal software. All parameters (number of contacts, number of dopaminergic fibers and neurons, number of contacts per neuron and the length of the contact) were quantified in 5 dissectors (sample fields) per single layer of the 20$\mu \mathrm{m}$-thick stacks. For each analysis, 8 stacks per brain region per animal were analyzed. The final result for the length of the contact was the average of at least 150 events per animal.

\section{Statistical analysis}

The data are expressed as the mean \pm SEM. A statistical analysis was performed using a student's t-test or one-way ANOVA test followed by a post hoc analysis (Tukey's test). The null hypothesis was rejected for an $\alpha$ risk equal to $5 \%$.

\section{Results}

\subsection{There is an increase in microglial polarity in chronic Parkinsonian macaques}

To understand whether microglial polarization and the formation of engulfing gliapses takes place in chronic Parkinsonian primates, we analyzed the brain sections of macaques with stable Parkinsonism by applying techniques and imaging protocols that were previously used in mice (Barcia et al., 2012). The primates were analyzed years after MPTP treatment. The results indicate that macaques with Parkinsonism show loss of dopaminergic neurons in the SNpc and a persistent increase in microglial activation (Fig. 1A-C), which has been the focus of recent extended studies in our group (Barcia et al., 2011). Although the number of Iba- $1^{+}$microglial cells did not change in the SNpc of Parkinsonian monkeys compared to control animals, the microglia clearly showed an activated phenotype, which was characterized by an increased cell body size and an increased number of terminal tips (Fig. 1D-E).

Because this increase in terminal tips may reflect the polarization of microglial cells toward damaged neurons, we analyzed the anatomical relationship between microglia and neurons in the SNpc of chronic Parkinsonian monkeys. Strikingly, years after the induction of Parkinsonism, microglial cells remain in a polarized state toward neighboring dopaminergic neurons when compared to those of control animals. A detailed confocal analysis indicated that there were an increased number of contacts between microglia and the remaining dopaminergic neurons in the SNpc years after the last MPTP insult. The analysis of the entire SNpc showed a prominent increase of interactions between microglial filopodia and dopaminergic structures (Fig. 2A and B). However, the contacts between microglial cell bodies and dopaminergic neurons are similar to those of the controls. Importantly, an analysis of particular sub-regions of the SNpc revealed that in Parkinsonian macaques, the number of body-to-body gliapse-like contacts remained higher than controls only in the ventral area of the SNpc, which is an area that is known to undergo the highest loss of dopaminergic neurons in Parkinsonian primates (Fig. 2C-E). In addition, the area of contact between microglia and dopaminergic neurons, including the neurites and neuronal bodies, was also increased in Parkinsonian monkeys (Fig. 2F).

\subsection{Microglia show persistent phagocytic characteristics}

A confocal analysis of the micro-anatomical characteristics of microglial cells in the SNpc of Parkinsonian macaques revealed that microglia exhibit phagocytic characteristics in the areas of neurodegeneration. One of the key elements, observed previously in the mouse MPTP-induced model, is an increase of Golgi apparatus protein machinery in microglial cells. This increase indicates that the microglia in the degenerating areas contain phagocytic and secretory functionalities. In macaques, we found that there is an increase in Golgi apparatus protein machinery, as indicated by GM130 staining, in Parkinsonian macaques compared to control animals (Fig. $3 \mathrm{~A}-\mathrm{C}$ ). This increase observed in the SNpc suggests that microglial cells remain in a persistent phagocytic state in chronic Parkinsonism. Further micro-anatomical analyses indicate that microglial cells contain numerous phagocytic buds in the SNpc of Parkinsonian monkeys (Fig. 3D-F and Supplementary Movies 1 and 2). These structures are rounded enlargements located at the microglial branches and display a ball-and-chain-like structure (Fig. 3D). These structures are reminiscent of the phagocytic events responsible for the elimination of apoptotic newborn cells in the adult mouse hippocampus (Sierra et al., 2010). In addition, a zoom-in confocal analysis indicated that phagocytic buds specifically contain GM130-expressing cisternae (Fig. 3E). Finally, three-dimensional reconstructions revealed the existence of engulfing gliapses in the SNpc of Parkinsonian monkeys 
A
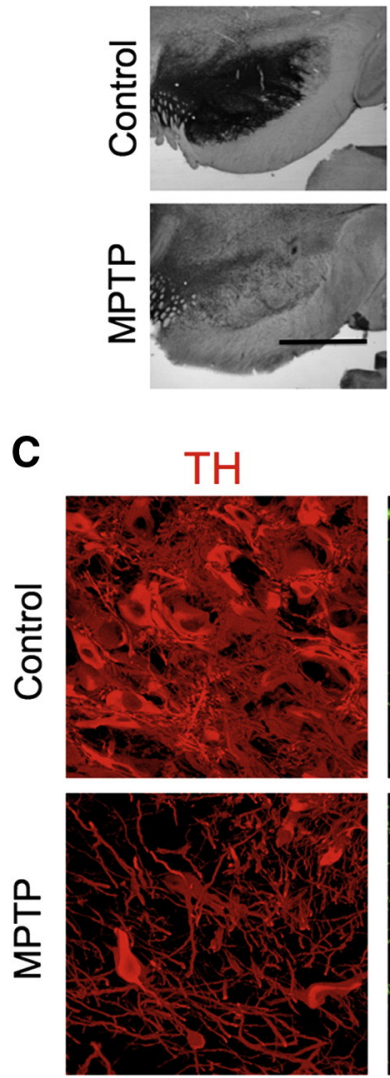

D
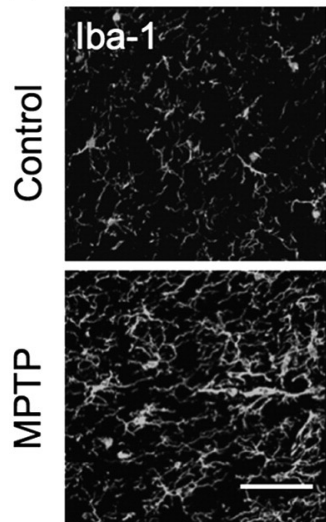

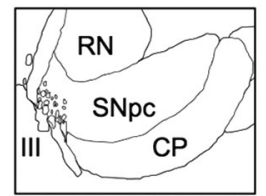

B

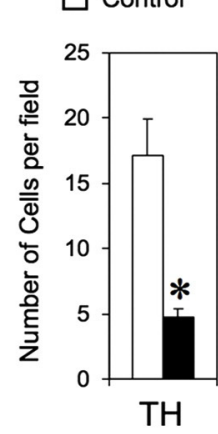

MPTP

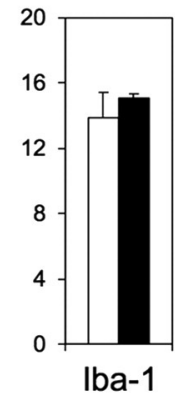

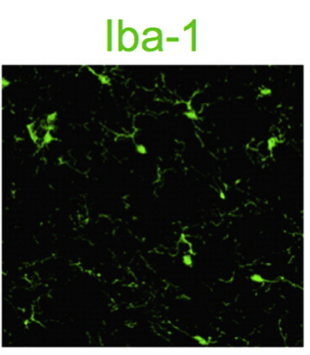
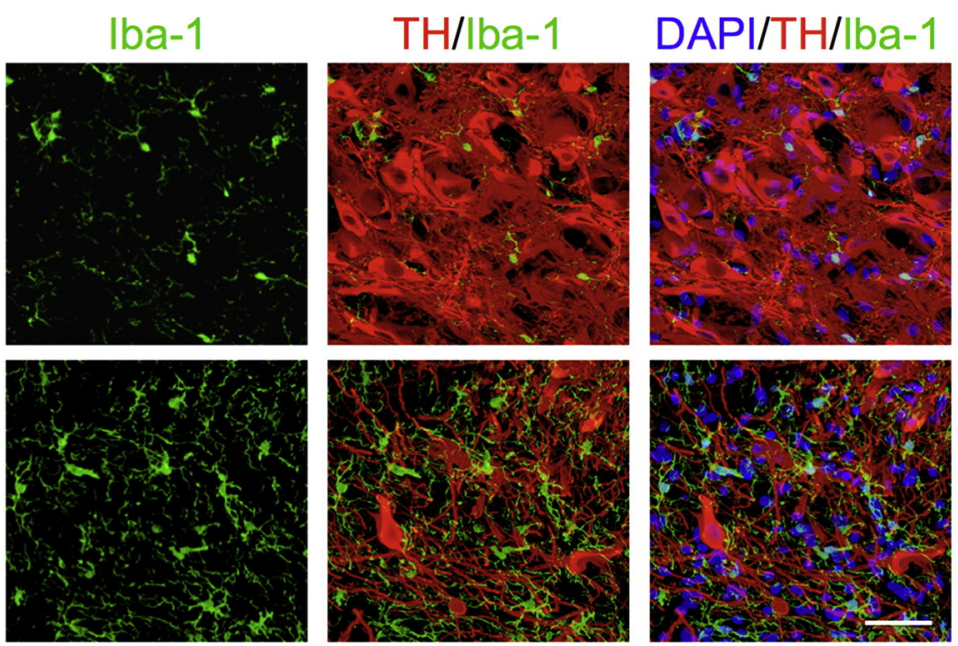

E

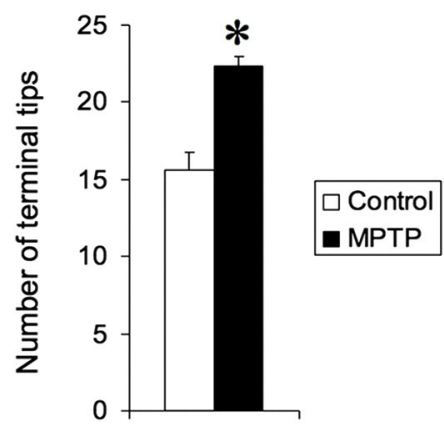

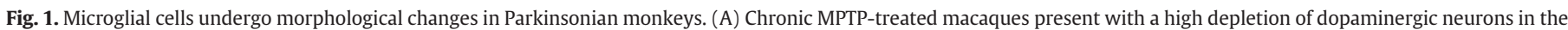

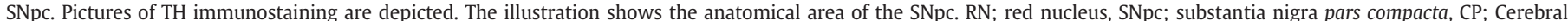

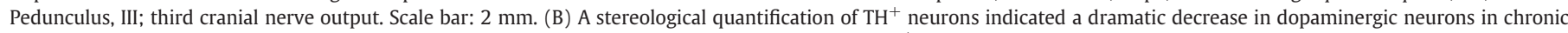

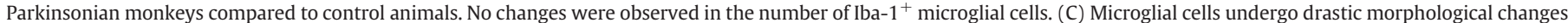

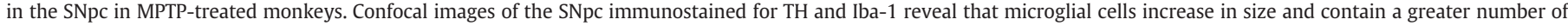

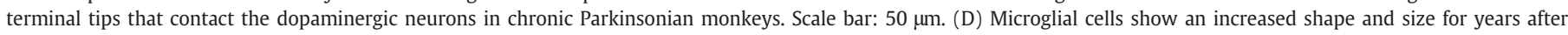

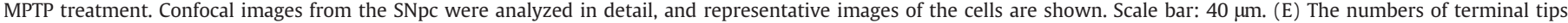
of microglial cells are significantly increased in MPTP treated monkeys. ${ }^{*} \mathrm{p}<0.05$ (student's t-test).

(Fig. 4). These structures were characterized by the engulfment of a pycnotic nucleus by a microglial cell, suggesting an ongoing degenerative process (Fig. 4). Three-dimensional reconstructions revealed that the microglial Golgi apparatus also surrounds the engulfed nucleus, suggesting an active secretory and phagocytic domain.

\section{Discussion}

In this study, we show that microglial cells maintain phagocytic characteristics in the areas of the brain where the degeneration of dopaminergic neurons takes place in chronic Parkinsonian monkeys years after MPTP insult. These microglial cells also possess an ongoing polarization of motility towards the degenerating neurons. These results indicate that microglia phagocytize dying dopaminergic cell bodies years after treatment with a neurotoxin, which is consistent with the well-established idea that there is a long term activation of microglial cells in Parkinsonian patients.

Contacts made between microglial and neuronal cell bodies, which are defined as gliapses, are significantly increased only in the ventral SNpc, which is the area with the highest vulnerability to 
A
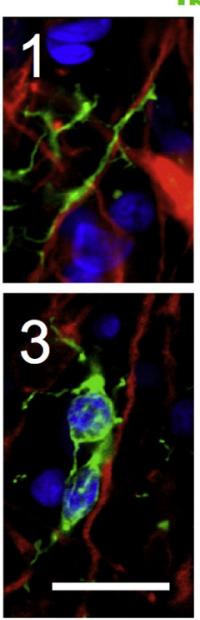

|lba-1/TH/DAPI

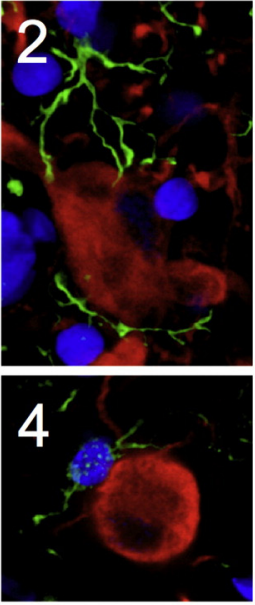

C
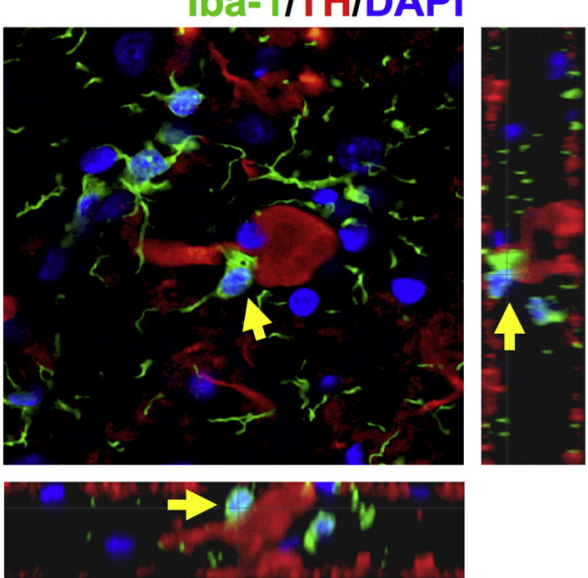

D Iba-1/TH/DAPI

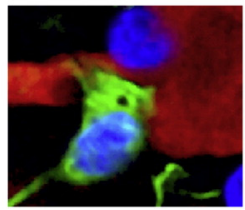

B
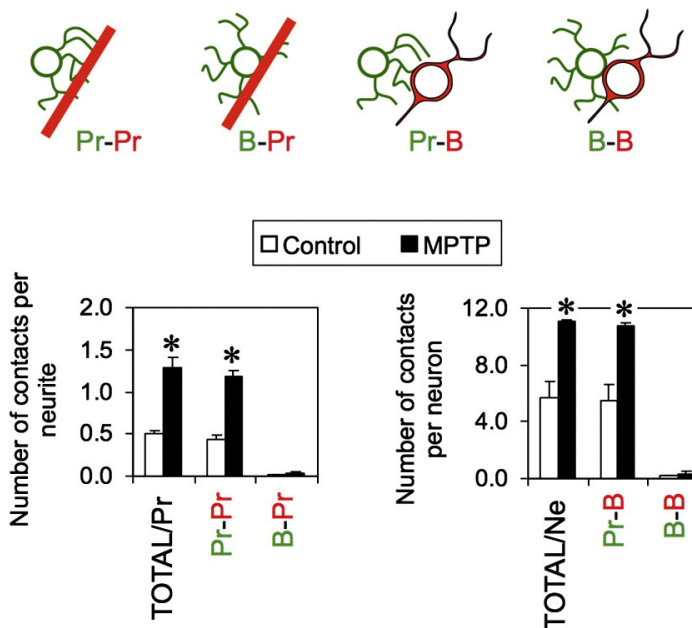

E
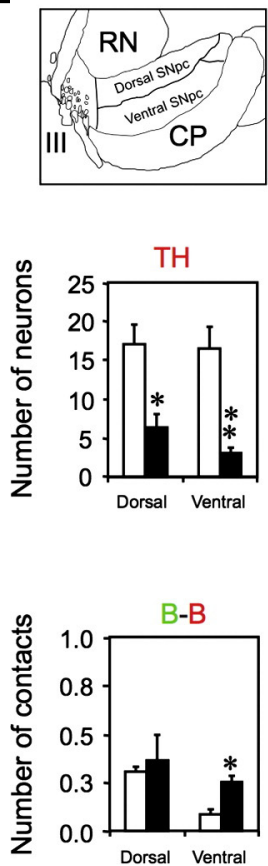

$\mathbf{F}$
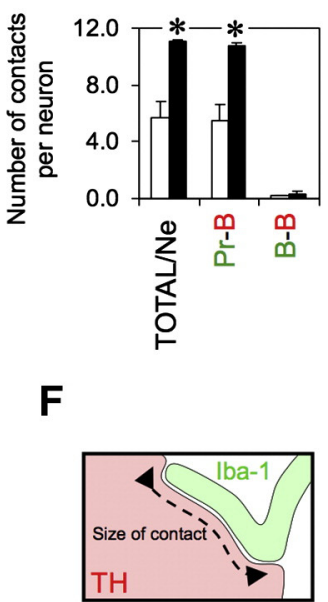

Control

MPTP

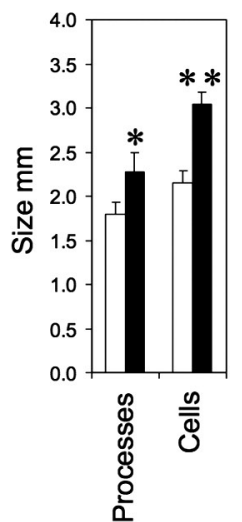

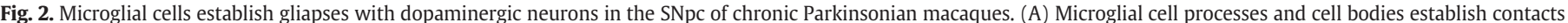

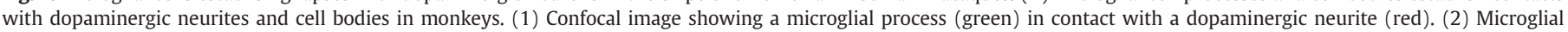

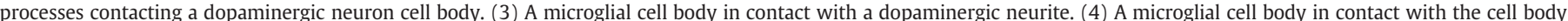

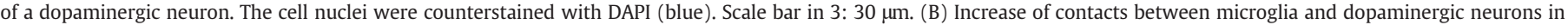

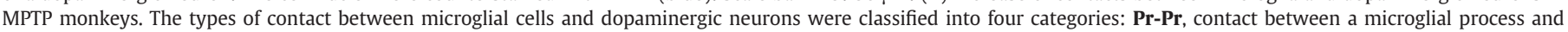

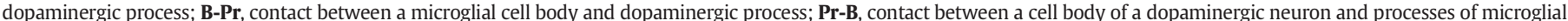

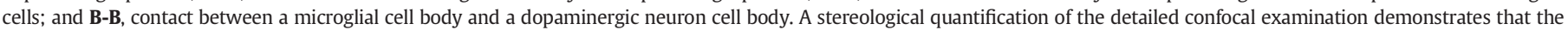

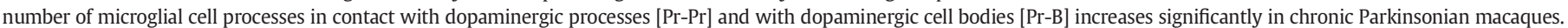

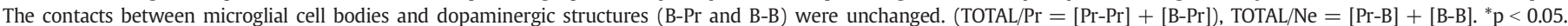

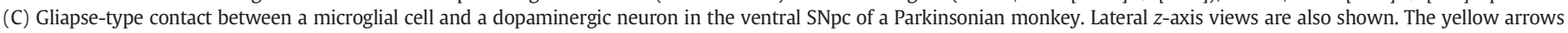

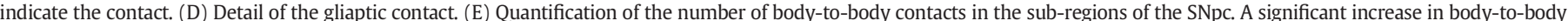

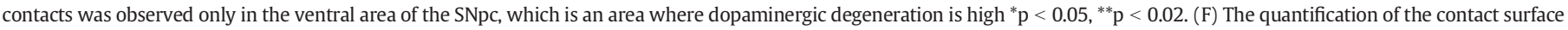
between microglial processes and dopaminergic neurons, either neurites or dopaminergic cell bodies, increases in chronic Parkinsonian macaques. * $\mathrm{p}<0.05$; ** $<0.01$.

MPTP. It is thought that the vulnerability of dopaminergic neurons of this area is due to many factors such as the levels of neuromelanin and calbindin (Yamada et al., 1990; Gibb and Lees, 1991). Thus, the present results indicate that there is an association between neuronal damage and gliapse formation, which suggest that microglial phagocytosis is an important event in Parkinsonian degeneration especially in vulnerable regions.
These microglial characteristics are specific to primates because the results found in macaques are in contrast with previous results obtained in mice (Barcia et al., 2012). In monkeys, the physical contact between microglial cells and neurons persists throughout the course of the disease, while in mice, cell-to-cell contact between microglia and neurons is transient and precedes neuronal elimination (Barcia et al., 2012). In the mouse model of PD, the engulfing process 
A

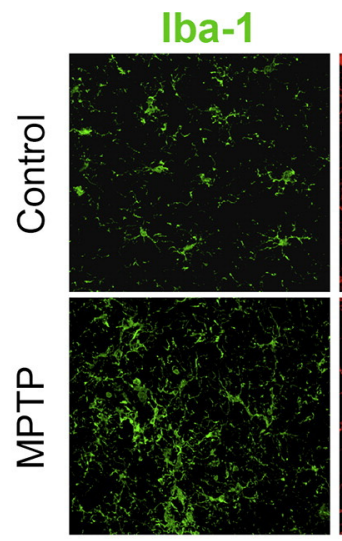

B
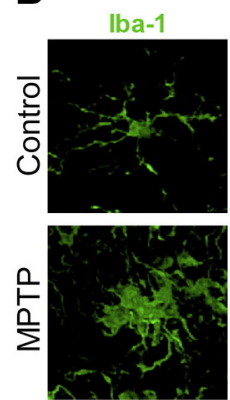
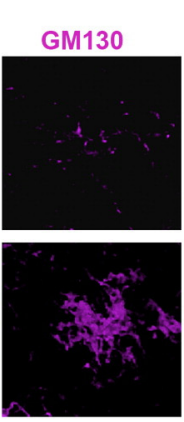

$\mathrm{TH}$
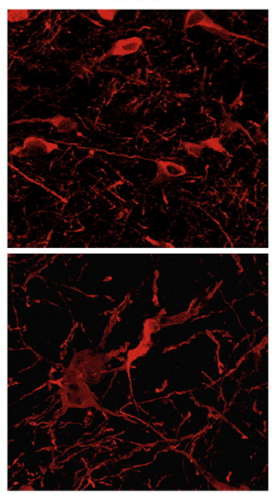

E
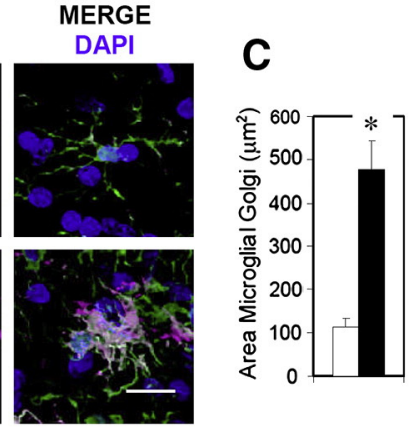

D

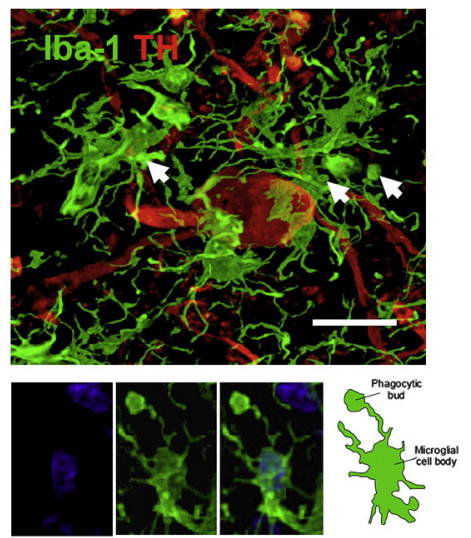

|lba-1/GM130

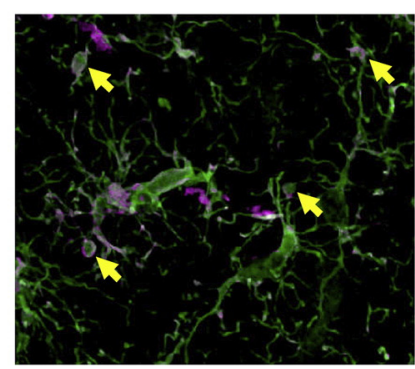

|lba-1/GM130/TH

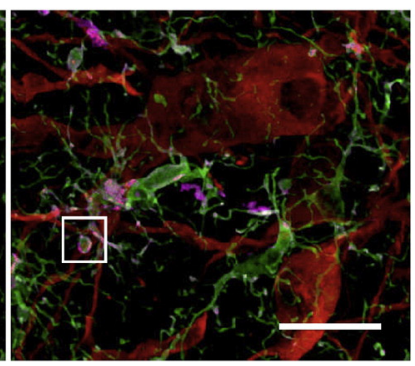

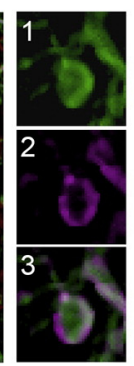

MERGE/DAPI
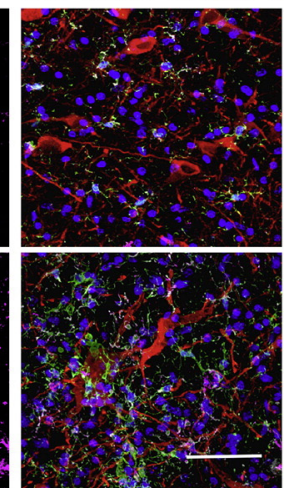
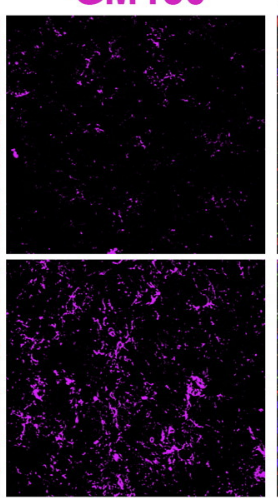

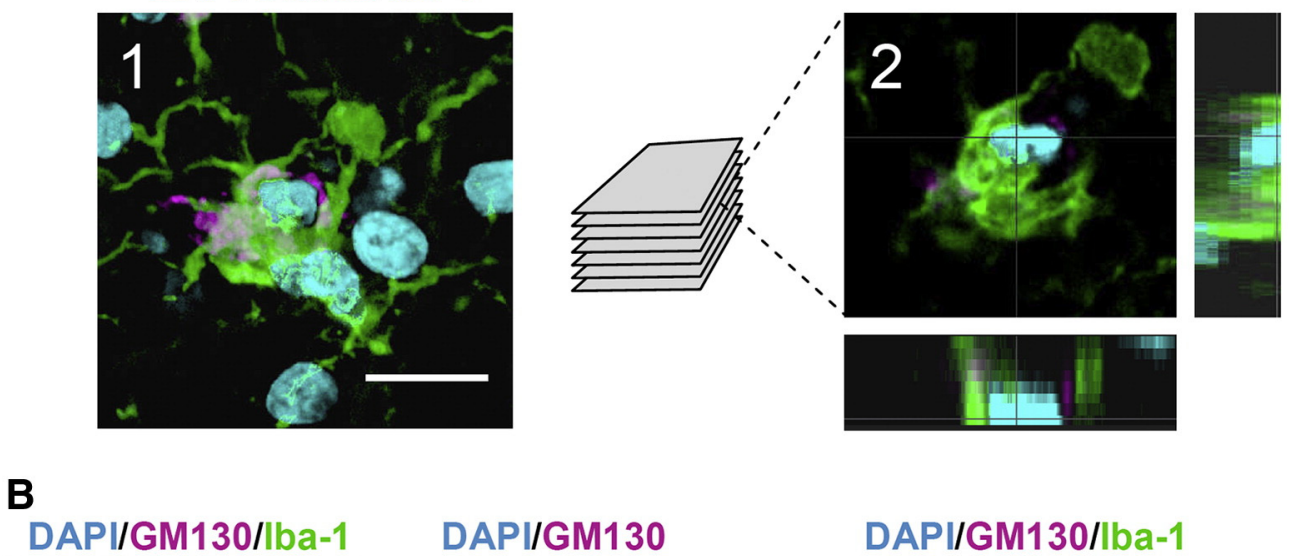

DAPI/GM130

DAPI/GM130/lba-1
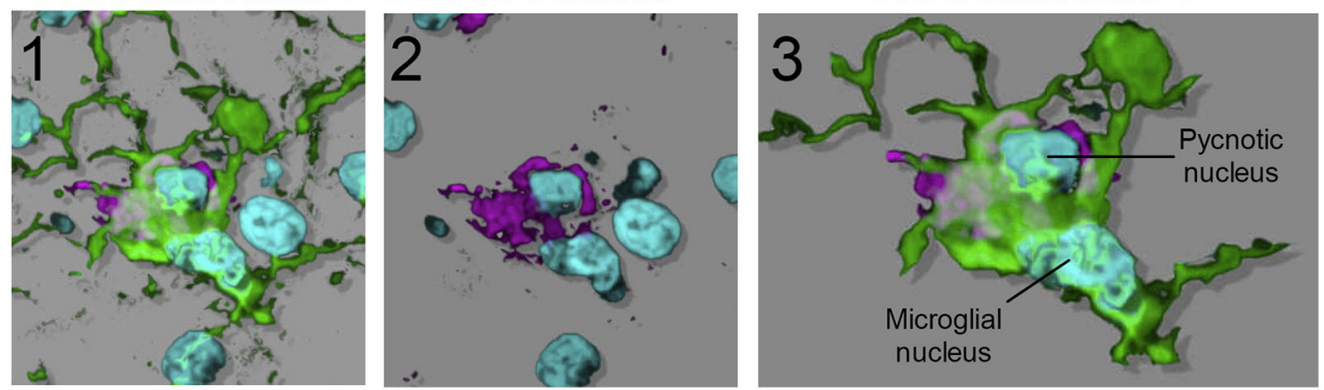

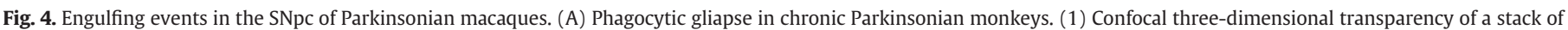

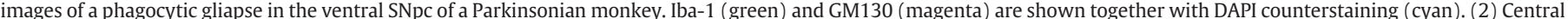

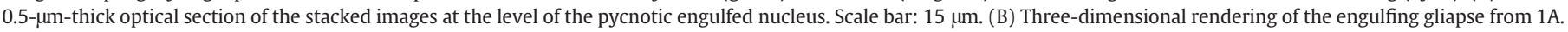

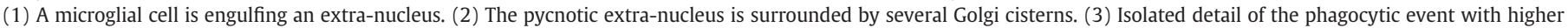
magnification.

Supplementary data to this article can be found online at http:// dx.doi.org/10.1016/j.jneuroim.2013.05.001.

\section{Acknowledgments}

This work was supported by grants from the Spanish Ministry of Science (SAF2010-21274, SAF2007-062262, FIS PI10-02827 and RYC2010-06729), Fundación Séneca (FS/15329/PI/10) and CIBERNED (Centro de Investigación Biomédica en Red sobre Enfermedades Neurodegenerativas). We would like to thank all of the personnel from the SAI (Servicio de Apoyo a la Investigación) for the help they provided at the University of Murcia, especially María García. Finally, the authors declare that there is no conflict of interest related to the present work.

\section{References}

Barcia, C., Bautista, V., Sanchez-Bahillo, A., Fernandez-Villalba, E., Navarro-Ruis, J.M., Barreiro, A.F., et al., 2003. Circadian determinations of cortisol, prolactin and melatonin in chronic methyl-phenyl-tetrahydropyridine-treated monkeys. Neuroendocrinology 78, 118-128.

Barcia, C., Sanchez Bahillo, A., Fernandez-Villalba, E., Bautista, V., Poza, Y.P.M., Fernandez-Barreiro, A., et al., 2004. Evidence of active microglia in substantia nigra pars compacta of Parkinsonian monkeys 1 year after MPTP exposure. Glia 46, 402-409.

Barcia, C., Ros, C.M., Annese, V., Gomez, A., Ros-Bernal, F., Aguado-Yera, D., et al., 2011. IFN-gamma signaling, with the synergistic contribution of TNF-alpha, mediates cell specific microglial and astroglial activation in experimental models of Parkinson's disease. Cell Death Dis. 2, e142.

Barcia, C., Ros, C.M., Annese, V., Carrillo-de Sauvage, M.A., Ros-Bernal, F., Gomez, A., et al., 2012. ROCK/Cdc42-mediated microglial motility and gliapse formation lead to phagocytosis of degenerating dopaminergic neurons in vivo. Sci. Rep. 2, 809.

Czlonkowska, A., Kohutnicka, M., Kurkowska-Jastrzebska, I., Czlonkowski, A., 1996. Microglial reaction in MPTP (1-methyl-4-phenyl-1,2,3,6-tetrahydropyridine) induced Parkinson's disease mice model. Neurodegeneration 5, 137-143.

Damier, P., Hirsch, E.C., Agid, Y., Graybiel, A.M., 1999. The substantia nigra of the human brain. II. Patterns of loss of dopamine-containing neurons in Parkinson's disease. Brain 122 (Pt. 8), 1437-1448.
Depboylu, C., Stricker, S., Ghobril, J.P., Oertel, W.H., Priller, J., Hoglinge, G.U., 2012. Brain-resident microglia predominate over infiltrating myeloid cells in activation, phagocytosis and interaction with T-lymphocytes in the MPTP mouse model of Parkinson disease. Exp. Neurol. 238, 183-191.

Gibb, W.R., Lees, A.J., 1991. Anatomy, pigmentation, ventral and dorsal subpopulations of the substantia nigra, and differential cell death in Parkinson's disease. J. Neurol. Neurosurg. Psychiatry 54, 388-396.

Gundersen, H.J., 1986. Stereology of arbitrary particles. A review of unbiased number and size estimators and the presentation of some new ones, in memory of William R. Thompson. J. Microsc. 143, 3-45.

Herrero, M.T., Hirsch, E.C., Kastner, A., Ruberg, M., Luquin, M.R., Laguna, J., et al., 1993. Does neuromelanin contribute to the vulnerability of catecholaminergic neurons in monkeys intoxicated with MPTP? Neuroscience 56, 499-511.

Kreutzberg, G.W., 1996. Microglia: a sensor for pathological events in the CNS. Trends Neurosci. 19, 312-318.

Langston, J.W., Forno, L.S., Tetrud, J., Reeves, A.G., Kaplan, J.A., Karluk, D., 1999. Evidence of active nerve cell degeneration in the substantia nigra of humans years after 1methyl-4-phenyl-1,2,3,6-tetrahydropyridine exposure. Ann. Neurol. 46, 598-605.

McGeer, P.L., Itagaki, S., Boyes, B.E., McGeer, E.G., 1988. Reactive microglia are positive for HLA-DR in the substantia nigra of Parkinson's and Alzheimer's disease brains. Neurology 38, 1285-1291.

McGeer, P.L., Schwab, C., Parent, A., Doudet, D., 2003. Presence of reactive microglia in monkey substantia nigra years after 1-methyl-4-phenyl-1,2,3,6-tetrahydropyridine administration. Ann. Neurol. 54, 599-604.

Nimmerjahn, A., Kirchhoff, F., Helmchen, F., 2005. Resting microglial cells are highly dynamic surveillants of brain parenchyma in vivo. Science 308, 1314-1318.

Olzewski, J., Baxter, D., 1954. Cytoarchitecture of the human brainstem. S. Karger by J. B. Lippincott Company, Philadelphia and Montreal.

Paxinos, G., Xu-Feng, H., Toga, A., 1999. The Rhesus Monkey Brain in Stereotaxic Coordinates. Academic Press, San Diego.

Sierra, A., Encinas, J.M., Deudero, J.J., Chancey, J.H., Enikolopov, G., Overstreet-Wadiche, L.S., et al., 2010. Microglia shape adult hippocampal neurogenesis through apoptosis-coupled phagocytosis. Cell Stem Cell 7, 483-495.

Vazquez-Claverie, M., Garrido-Gil, P., San Sebastian, W., Izal-Azcarate, A., Belzunegui, S., Marcilla, I., et al., 2009. Acute and chronic 1-methyl-4-phenyl1,2,3,6-tetrahydropyridine administrations elicit similar microglial activation in the substantia nigra of monkeys. J. Neuropathol. Exp. Neurol. 68, 977-984.

Yamada, T., McGeer, P., Baimbridge, K., McGeer, E., 1990. Relative sparing in Parkinson's disease of substantia nigra dopamine neurons containing calbindin-D28K. Brain Res. 526, 303-307. 